\title{
Physics Potentials of the Hyper-Kamiokande Second Detector in Korea
}

\author{
Seon-Hee Seo*† \\ Center for Underground Physics, Institute for Basic Science, Daejeon, 34126, S. Korea \\ E-mail: sunny.seo@ibs.re.kr
}

\begin{abstract}
Hyper-Kamiokande (Hyper-K) succeeds the very successful Super-K experiment and will consist of a large detector filled with 260 kton purified water and equipped with $40 \%$ photo-coverage. Physics program of Hyper-K is broad, covering from particle physics to astrophysics and astronomy. The Hyper-K $1^{\text {st }}$ detector will be built in Japan, and the $2^{\text {nd }}$ detector is considered to be built in Korea because locating the $2^{\text {nd }}$ detector in Korea improves physics sensitivities in most cases thanks to the longer baseline $(\sim 1,100 \mathrm{~km})$ and larger overburden $(\sim 1,000 \mathrm{~m})$ for Korean candidate sites. In this talk, we present overview and physics potentials of the Hyper-K $2^{\text {nd }}$ detector in Korea.
\end{abstract}

The 39th International Conference on High Energy Physics (ICHEP2018)

4-11 July, 2018

Seoul, Korea

\footnotetext{
* Speaker.

${ }^{\dagger}$ This work was supported by the National Research Foundation of Korea (NRF) grant funded by the Korea government (MSIT) (No. 2017R1A2B4012757 and IBS-R016-D1-2018-b01).
} 


\section{Introduction}

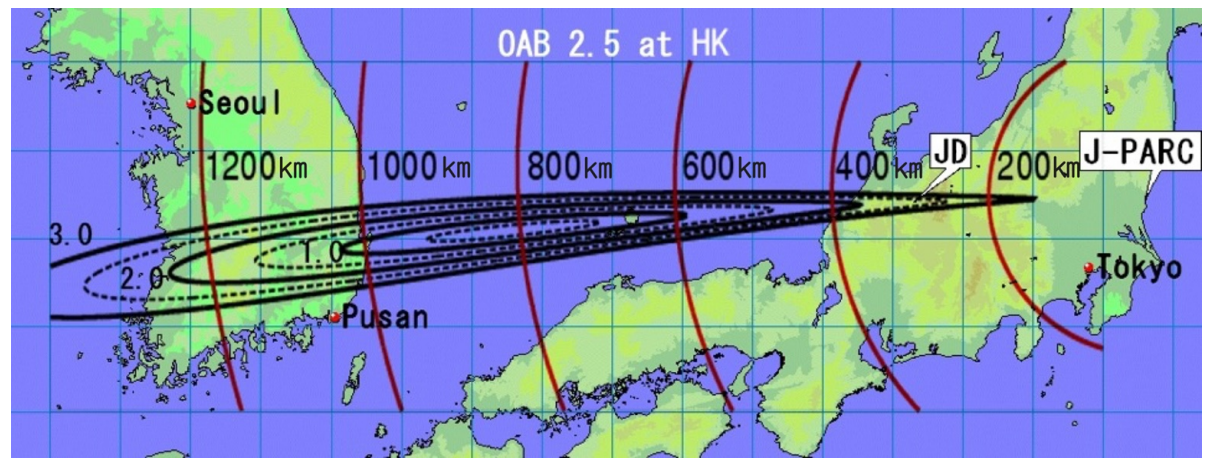

Figure 1: Baselines and off-axis angles of the J-PARC neutrino beam in Japan and Korea [2].

Hyper-Kamiokande (Hyper-K) is a next generation water Cherenkov detector consisting of two identical detectors with each 260 kton purified water and $40 \%$ photo coverage with a combination of 20 inch Hamamatsu PMTs and multi-PMTs (multiple 3 inch PMTs in one enclosure). Hyper-K will start its construction for the $1^{\text {st }}$ detector [1] in April 2020 at Tochibora site where the baseline from J-PARC is $295 \mathrm{~km}$ with $2.5^{\circ}$ off-axis angle (OAA).

The neutrino beam produced at J-PARC currently reaches Korea with $1^{\circ} \sim 3^{\circ}$ OAA from $1,000 \mathrm{~km}$ to $1,260 \mathrm{~km}$ baselines (see Fig. 1). This gives an opportunity to locate the $2^{\text {nd }}$ detector in Korea since the longer baseline would benefit various physics potentials such as precise measurement of $\mathrm{CP}$ violation phase and neutrino mass ordering determination. Bi-probability plots in Fig. 2 clearly shows that it is easier to determine neutrino mass ordering and $\mathrm{CP}$ violation phase in a Korean Mt. Bisul site. This is due to the longer baseline and higher energy reach from the $1^{\text {st }}$ and $2^{\text {nd }}$ oscillation maxima. Table 1 shows two most favorable candidate sites in Korea. Thanks to larger overburden of $\sim 1000 \mathrm{~m}$ in Korean candidate sites than Japan Tochibora site $(650 \mathrm{~m})$, low energy physics sensitivities such as solar neutrinos, supernova relic neutrinos are expected to be improved. Due to a very limited space here we present only some physics sensitivities, but more

Table 1: Two favorable sites of the Hyper-K $2^{\text {nd }}$ detector in Korea with off-axis angles between $1^{\circ}$ and $2.5^{\circ}$. The baseline is the distance from the production point of the J-PARC neutrino beam to the candidate sites [3].

\begin{tabular}{lcccl}
\hline \hline Site & Off-axis angle & Baseline $(\mathrm{km})$ & Height $(\mathrm{m})$ & Composition of rock \\
\hline Mt. Bisul & $1.3^{\circ}$ & 1,088 & 1,084 & Granite porphyry, andesitic breccia \\
Mt. Bohyun & $2.3^{\circ}$ & 1,043 & 1,124 & Granite, volcanic rocks, volcanic breccia \\
\hline \hline
\end{tabular}

details are found in [3].

\section{Physics potentials}

Sensitivity studies are performed using NEUT [4] 5.3.2 for neutrino interaction generator and full simulation of Super-K scaled to Hyper-K for the expected event rates, Prob3++ [5] for the 

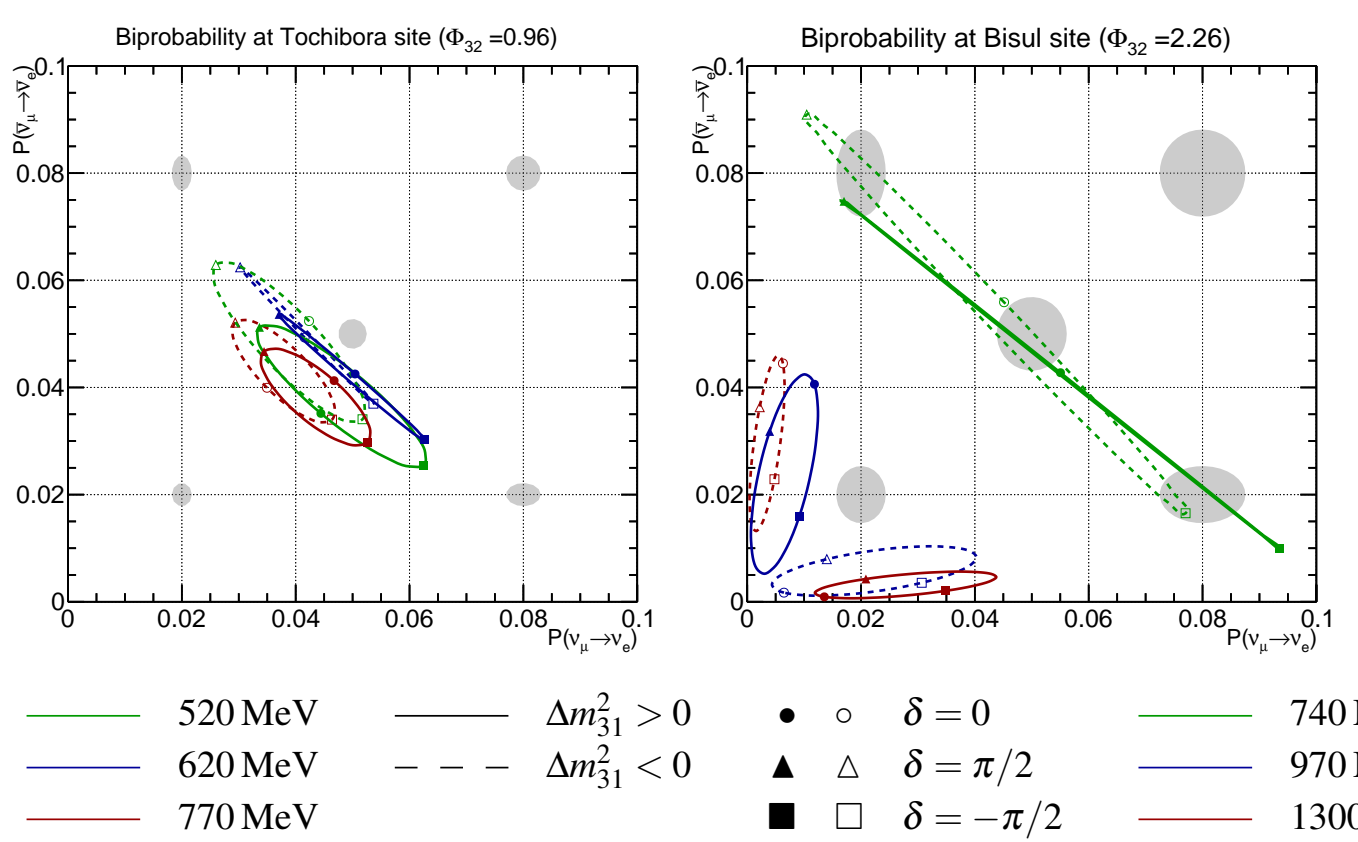

$$
\begin{aligned}
& \text { - } \circ \delta=0 \\
& \Delta \triangle \delta=\pi / 2 \quad-970 \mathrm{MeV} \\
& \square \quad \square=-\pi / 2
\end{aligned}
$$

Figure 2: $v_{e}$ appearance bi-probabilities at the Hyper-K site in Tochibora, Japan (left) and Mt. Bisul, Korea (right). Grey ellipses show the sizes of statistical uncertainties for a ten year exposure of one Hyper-K detector, and $\Phi_{32} \equiv \frac{2}{\pi} \frac{\left|\Delta m_{32}^{2}\right| L}{4 E}$ where $\left|\Delta m_{32}^{2}\right|=2.5 \times 10^{-3} \mathrm{eV}^{2}, E=620 \mathrm{MeV}$ (left), and $970 \mathrm{MeV}$ (right) [3].

oscillation probabilities, and a constant matter density of $3.0 \mathrm{~g} / \mathrm{cm}^{2}$ for a $1100 \mathrm{~km}$ baseline with $1.5^{\circ}, 2.0^{\circ}$, or $2.5^{\circ} \mathrm{OAA}$ for hypothetical Korean sites.

In our sensitivity studies, unless otherwise specified, 10 years of operation with $1.3 \mathrm{MW}$ beam power is assumed with 1:3 ratio of neutrino to antineutrino modes, and this corresponds to $2.7 \times$ $10^{22}$ proton on target (POT). A reasonable systematic uncertainty model is also applied [3].

According to our sensitivity studies, when the $\mathrm{CP}$ is maximally violated with known mass ordering, there is almost no difference in the CP violation sensitivity between Japan Detector (JD) plus Korean Detector $(\mathrm{KD})$ configuration and two Japan Detectors $(\mathrm{JD} \times 2)$. However, when the $\mathrm{CP}$ is a little non-maximally violated JD $+\mathrm{KD}$ sensitivity is better.

Figure 3 left plot shows the progress of $\delta_{c p}$ violation discovery potential (minimum $5 \sigma$ significance) with exposure for known mass ordering. Due to the limited statistics, the sensitivity of the first 4.5 years is worse for any JD $+\mathrm{KD}$ configuration than JD $\times 2$. After then any JD $+\mathrm{KD}$ configuration sensitivity gets better and reaches close to $\sim 60 \%$ fraction of $\delta_{c p}$, while $\sim 55 \%$ for JD $\times 2$ limited by systematic uncertainties. The aim of Hyper-K is not only to determine $\mathrm{CP}$ violation or not but also precise measurement of $\delta_{c p}$ value. By locating the $2^{\text {nd }}$ detector in Korea we can improve this sensitivity and this is an important input for flavor symmetry models. Figure 3 right plot shows the $1 \sigma$ precision of the $\delta_{c p}$ measurement as a function of the true $\delta_{c p}$ value with the mass ordering unknown for true normal ordering. When the $\mathrm{CP}$ is maximally violated cases, the precision changes from $\sim 22^{\circ}(\mathrm{JD} \times 1)$ and $\sim 17^{\circ}(\mathrm{JD} \times 2)$ to $13 \sim 14^{\circ}\left(\mathrm{JD}+\mathrm{KD}\right.$ at $\left.1.5^{\circ} \mathrm{OAA}\right)$.

Sensitivity to determine mass ordering is improved with JD+KD configuration. Atmospheric neutrinos add additional increase of the sensitivity. Figure 4 shows wrong mass ordering rejection significance as a function of different octant values for true normal ordering case. For any $\sin ^{2} \theta_{23}$ 

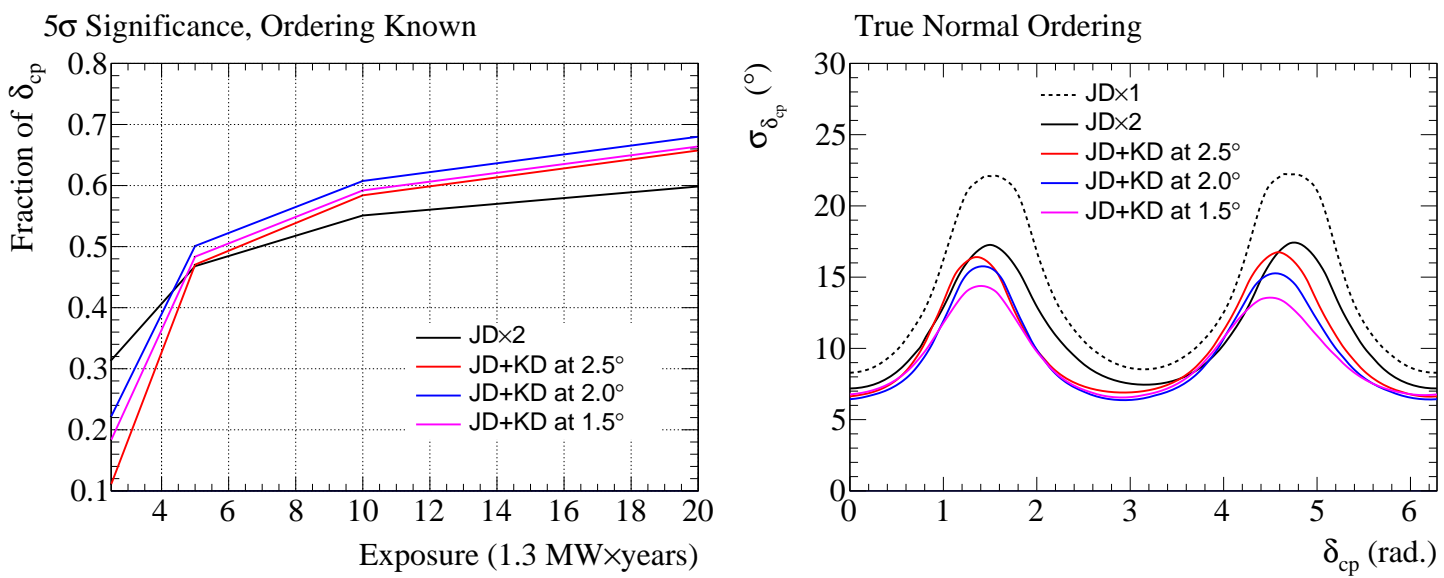

Figure 3: (left) The fraction of $\delta_{c p}$ values (averaging over the true mass ordering) to reject the CP conserving values of $\delta_{c p}$ with at least a $5 \sigma$ significance for known mass ordering. (right) The $1 \sigma$ precision of the $\delta_{c p}$ measurement as a function of the true $\delta_{c p}$ value with unknown mass ordering for true normal ordering [3].

value between 0.4 and 0.6 , more than $8 \sigma$ sensitivity is expected for JD+KD (Mt. Bisul) configuration when the atmospheric neutrinos are combined with beam neutrinos.

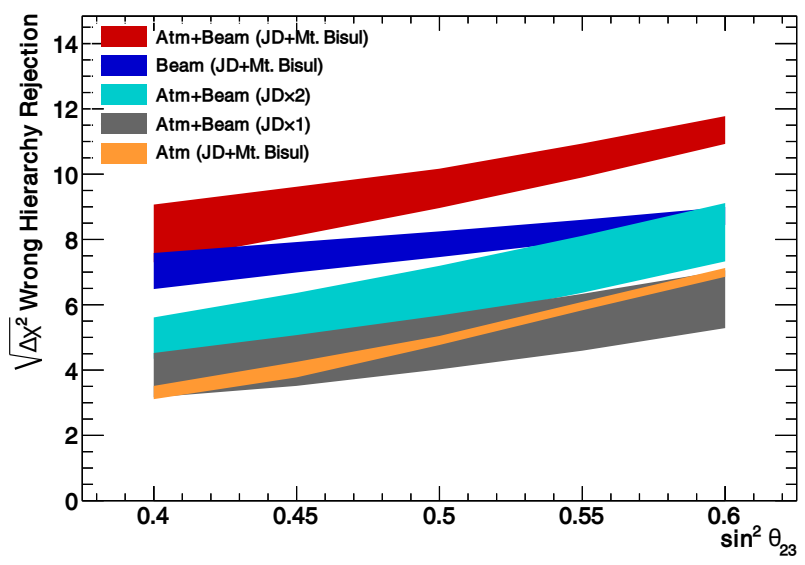

Figure 4: Sensitivity to reject wrong mass ordering assuming true normal ordering with a combined measurement of beam and atmospheric neutrinos for a 10 year exposure. The $\mathrm{x}$-axis shows the possible ranges of $\sin ^{2} \theta_{23}$ and the width of the bands comes from the variation in sensitivity with $\delta_{c p}$ [3].

\section{References}

[1] K. Abe et. al. (Hyper-Kamiokande Proto-Collaboration), arXiv:1805.04163[hep-ex].

[2] K. Hagiwara, N. Okamura, K. Senda, Phys. Lett. B 637, 266 (2006), Erratum: Phys. Lett. B 641, 491 (2006); K. Hagiwara, N. Okamura, K. Senda, Phys. Rev. D 76, 093002 (2007).

[3] K. Abe et. al. (Hyper-K Proto-Collab.), PTEP 2018, 063 C01 (2018); arXiv:1611.06118[hep-ex].

[4] Y. Hayato, Acta Phys. Polon. B 40, 2477 (2009).

[5] http://webhome.phy.duke.edu/ raw22/public/Prob3++/ 